\title{
Mechanical Analysis of Ship Borne Maneuvering and Improvement of Cable Arresting Device
}

\author{
Wenkui Ma \\ School of North China Electric Power University, Baoding 071000, China \\ 1663476907@qq.com
}

Keywords: Arresting Cable, Stress Analysis, Motion Equation

\begin{abstract}
In this paper, the aircraft is the main research object. Using CAD rendering model diagram and force analysis diagram, we analyzed the dynamic model in arresting cable under the action of the landing, and got the general rule of aircraft and arresting cable force based on theoretical mechanics knowledge. Then we demonstrated the rule by a specific analysis model of ship borne a movement of ship to determine the relevant parameters and the corresponding blocking cable materials, to provide reference for the research of aircraft; With the analysis of the existing law, the aircraft arresting landing causing the accident of the aircraft arresting gear in the past is improved.
\end{abstract}

\section{Introduction}

Aircraft carrier to stop the cable, used to stop the carrier based aircraft high-speed, is the carrier of the real lifeline". As the last guarantee of the landing of carrier aircraft, the arresting cable has always been a difficult problem in the system. Research on the system and its related devices from the beginning of 1950s in foreign countries, November 25, 2012, China's first carrier based aircraft to block the ship test has been a complete success! However, there are still cable stop after the aircraft landing deck speed is too high, can not afford the huge tension cable stop tail hook moment, resulting in arresting cable fracture problems, At this time the carrier aircraft can not stop on the deck, often out of control, the accident occurred in the fall of the sea.

\section{Model Simplification and Motion Analysis of Carrier Based Aircraft}

It is a special dynamic problem that the deck motion is a special dynamic problem, and its stress condition is more complicated. In the process of stopping, the carrier aircraft and the runway on the blocking effect of the cable, To stop the rope through the track on both sides of the pulley block equipment to work, the aircraft's kinetic energy into heat energy, so as to achieve the purpose of reducing the aircraft in a short distance [1], as shown in Figure 1.

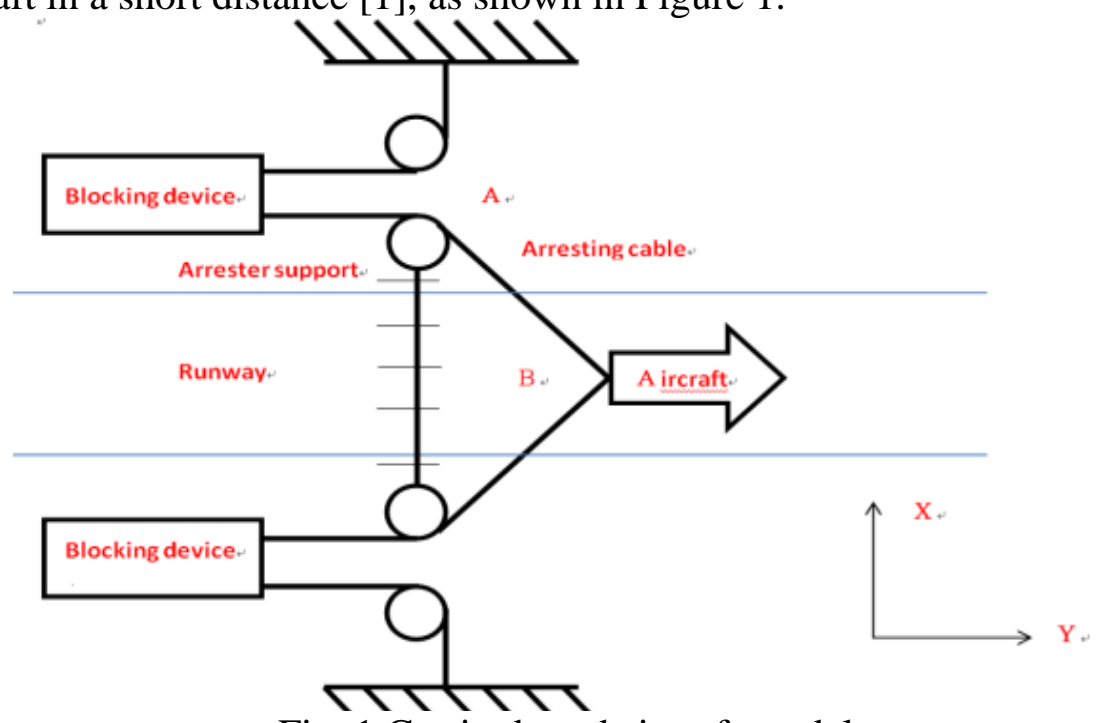

Fig. 1 Carrier based aircraft model 
Several Hypotheses in the Process of Model Simplification. Assumes that the carrier is not considering the effect of uniform motion, converted inertial force caused by the movement of the aircraft carrier.

Will approximate the aircraft and its ancillary equipment ideal rigid body, without considering its elastic deformation, the quality of the aircraft and its ancillary equipment is concentrated in the center of mass.

Takes the ground as a flat surface and ignores the curvature of the earth.

For takeoff and landing when the height from the ground, so that the acceleration of gravity and atmospheric density constant.

Force Analysis of Carrier Based Aircraft. The main force of carrier based on the action of arresting cable includes: Aircraft aerodynamic load $F_{\mathrm{q}}$, Aircraft and its ancillary equipment gravity $G$. The support force of the ground plane $F_{\mathrm{N}}$, Friction force $F_{\mathrm{f}}$, Arresting force $F$ and thrust of engine $F_{\mathrm{q}}$ [2]. Carrier aircraft landing in the block after the role of the cable under the specific circumstances such as the figure 2 below.

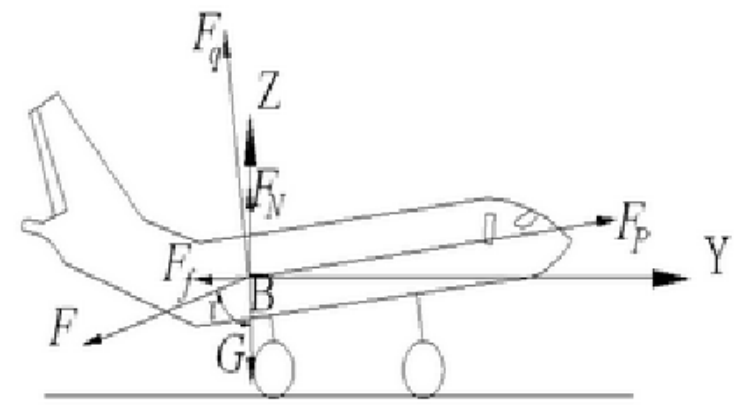

Fig. 2 Stress analysis of ship borne aircraft under the action of arresting cable

Dynamics Equation of Carrier Aircraft in ZBY Plane. Differential equations along the $Y$ axis:

$$
\frac{m d^{2} y}{d t^{2}}=F_{p y}-F_{f}-F_{q y}-F_{y}
$$

Equilibrium equation along $Z$ axis:

$$
F_{p z}+F_{N}+F_{q z}-G-F_{z}=0
$$

Aerodynamic load along the $Y$ axis of resistance:

$$
F_{q y}=1 / 2\left(\rho v_{\mathrm{s}}^{2} S C_{y}\right)
$$

Aerodynamic load along the $Z$ axis of resistance:

$$
F_{\mathrm{qz}}=1 / 2\left(\rho \mathrm{v}_{\mathrm{s}}^{2} S C_{\mathrm{z}}\right)
$$

Horizontal friction:

$$
F_{f}=\mu F_{N}
$$

The significance of each physical quantity in the formula: $M$-aircraft quality, $Y$-the distance along the runway after the flight, $F_{p}$-aircraft engine thrust $F_{p y}$-aircraft engine thrust in the $Y$ direction, $F_{f}$-deck friction, $F_{q}$-aerodynamic loads on the aircraft, $F_{q y}$-the aerodynamic load along the $Y$ axis of the aerodynamic load along the $F_{p z}$ axis, the thrust of the aircraft engine in the $Z$ direction, $P$-air density, $v_{\mathrm{s}}$ aircraft of the instantaneous velocity, $s$-wing area, $C_{y} C_{z}$ - aerodynamic coefficients, $F$-stop cable force of the aircraft. $F_{y} F_{z}$-stop cable on the aircraft in the $Z, Y$ direction of the force, $F_{N}$-to face the support of the aircraft, $F_{q y}$-the aerodynamic load along the $Z$ axis of the lift force, $G$-aircraft and its ancillary equipment gravity [3].

\section{The Calculation of the Equation and the Result Analysis.}

Also $F_{p y} F_{p z}$ and $F_{q z} \quad F_{q y} C_{y} C_{z}$ air carrier -- although the longitude and latitude, altitude height and carrier based aircraft of different types, according to the relevant instruments, the local density $\mathrm{p}$, aircraft instantaneous velocity $v_{\mathrm{s}}$ can be measured, the wing area $\mathrm{s}$ and aerodynamic coefficient 
were known carrier borne machine setting parameters, so by equations (3) and (4) can be regarded as the aerodynamic load along the $Y$ and $Z$ axis resistance; then the aircraft carrier deck, the friction coefficient mu known, carrier based aircraft engine along the $Y$ and $Z$ axis thrust (for multi engine is the engine of the resultant) aircraft known parameters, so the simultaneous equations (1), (2), (5) and (6) can be solved in arresting cable under the action of carrier based aircraft along the $Y$ axis motion displacement $y$ and the speed $V$ and block of carrier based aircraft along the $Y$ and $Z$ axis component of the dynamic relationship between the cable[4].

\section{Examples of Analysis}

Choose the United States aircraft carrier MK7-3 type block device, the aircraft carrier uniform level of travel, aircraft landing for the suspension cable, try to analyze the force of the aircraft to stop the cable.

Analysis: the assumption that early aircraft just and arresting cable contact velocity $V_{0}$, arbitrary velocity $V$, initial displacement is 0 , the displacement at any moment to $y$, carrier based aircraft along the $Y$ and $Z$ direction of the engine thrust is $F_{p y}, F_{p z}$ known, the friction coefficient mu, wing area s, air density rho of aerodynamic coefficients number $C_{y} C_{z}$ are known

$$
\frac{d^{2} y}{d t^{2}}=\frac{d v}{d t}=\frac{v d v}{d y}
$$

Using equations (2), (3), (4), (5), (6) and (1), a substitution can be obtained as follows.

$$
\frac{m v d v}{d y}=F_{p y}-\mu G-(\mu+1) F_{y}+\frac{(\mu-1)\left(\rho v_{s}^{2} S C_{y}\right)}{2}
$$

Both sides of the equation to be adjusted:

$$
\frac{m v d v}{F_{p y}-\mu G-(\mu+1) F_{y}+\frac{(\mu-1)\left(\rho v_{s}^{2} S C_{y}\right)}{2}}=d y
$$

Simultaneous integral on both sides of the equation:

$$
\int_{v o}^{v} \frac{m v d v}{F_{p y}-\mu G-(\mu+1) F_{y}+\frac{(\mu-1)\left(\rho v_{s}^{2} S C_{y}\right)}{2}}=\int_{0}^{y} d y
$$

Note: $V$ is the component of $v_{\mathrm{s}}$ in the $Y$ direction

Use on the aircraft at any time speed $V$ and displacement of substitution can be obtained aircraft stopped cable force is blocked in the $Y$ direction force $F_{y}$.

Then the simultaneous equations (2), (3), (4) and (5) can be obtained by force aircraft stopped blocked cable force along the $Z$ axis direction [5].

$$
F_{z}=F_{p z}+F_{N}+\frac{1}{2\left(\rho \mathrm{v}_{\mathrm{s}}^{2} S C_{z}\right)}
$$

Then according to the synthesis of force, the force of the carrier of the carrier:

$$
F=\sqrt{F_{y}^{2}+F_{z}^{2}}
$$

By the end of Newton's third law, you can get on the tail hook of carrier aircraft arresting cable force situation. The arresting cable can withstand the maximum tensile force to determine the relevant parameters.

\section{Conclusion}

Model simplification process may be too idealistic, ignoring the impact of some objective factors, resulting in a simplified model and the actual situation has a certain discrepancy. Due to factors such as knowledge level constraints, only with the carrier aircraft of analysis to obtain performance 
parameters of arresting cable, if you can to stop cable as the research object, aiming at the process of dynamic change by $X_{\text {treme }}$ program and ANSYS secondary development of procedures to establish the simulation model, simulation calculation and drawing, through the demonstration of the block cable in a plane shock loading force, directly derived should stress and strain graphics, and the result is more accurate.

\section{References}

[1]Ho C.M., Tai Y. C. Micro-electro-mechanical systems (MEMS) and fluid flows [J]. Ann. Rev. Fluid Mech. 1998(30): 579-612.

[2]Steudle E. The cohesion-tension mechanism and the acquisition of water by plant roots.

[3]Annual Review Plant Physiology Plant Molecular Biology, 2001, 52,847- 875.

[4]Sperry JS, Tyree MT. Mechanism of water stress-induced xylem embolism [J]. Plant Physiology, 1988, 88: 581-587.

[5] Huang Sheng, Meng Xiangyin, Chang Xin. The carrier aircraft landing prediction and Analysis on sliding distance, College of marine engineering, Harbin Engineering University, 2009:7-9 